\title{
Correlation of Coagulation Abnormalities and Severity of Disease by Computed Tomography in Indian COVID-19 patients
}

\author{
Bhandari $\mathrm{S}^{1,}$ Rankawat $\mathrm{G}^{2^{*}}$, Bagarhatta $\mathrm{M}^{3}$, Singh $\mathrm{A}^{4}$, Agrawal $\mathrm{A}^{5}$, Kumar $\mathrm{A}^{6}$ \\ 1. Senior professor, Department of General medicine, SMS Medical college and attached group of Hospital, Jaipur, Rajasthan, India. \\ 2. Resident/Fellow student, Department of General Medicine, SMS medical college, Jaipur, Rajasthan, India. \\ 3. Senior professor, Department of Radiodiagnosis, SMS Medical college and attached group of Hospital, Jaipur, Rajasthan, India. \\ 4. Senior specialist, Department of General medicine, SMS Medical college and attached group of Hospital, Jaipur, Rajasthan, India \\ 5. Senior professor, Department of General medicine, SMS Medical college and attached group of Hospital, Jaipur, Rajasthan, India. \\ 6. Senior Demonstrator, Department of Pathology, SMS Medical college and attached group of Hospital, Jaipur, Rajasthan, India.
}

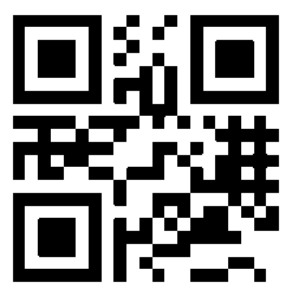

Abstract :

Background: The present study was undertaken to investigate and quantify the abnormal coagulable state in COVID-19 patients and to correlate it with the severity of COVID-19 infection as determined by high-resolution computed tomography (HRCT) of the chest. Materials and methods: In an attempt to provide extensive information pertaining to blood coagulation and radiological characteristics of COVID-19, the present study was undertaken in120 hospitalized patients with SARS CoV-2 infection confirmed positive by RT-PCR at SMS Hospital, Jaipur, India. For all patients, coagulation profile was evaluated in form of fibrin degradation products (FDP) and D-dimer, Theses values were then correlated with HRCT chest findings. Results: Among the confirmed cases, most of patients were young adults, in the fifth and sixth decade of age group with mean age of 50.40 years. There was a male preponderance (59\% male and $41 \%$ female). Out of total analyzed patients,59 patients $(49.16 \%)$ were symptomatic and55\% patients had some underlying co-morbid disease. Fifty-one patients (42.5\%) had raised FDP with mean value $17.9 \mu \mathrm{g} / \mathrm{mL}$ while sixty-six patients $(55 \%)$ had elevated D-dimer level with mean value $3.3 \mu \mathrm{g} / \mathrm{mL}$. We found $60.83 \%$ patients had CT findings classically described with COVID-19 infection. In $91.2 \%$ of those with normal FDP levels and $94.5 \%$ of those with D-dimer within normal levels, the CT severity score was found to be $<10 / 25$. CT severity score was found to be $>10 / 25$ in $78 \%$ and $68 \%$ patients respectively who had FDP and D-Dimer level more than four times of upper normal limits (UNL). Pearson correlation coefficient for FDP and CT severity score were found $r=+0.6494$ with $p$-value $<0.001$ while for $\mathrm{D}$-dimer and CT severity score $\mathrm{r}=+0.7143$ with $\mathrm{p}$-vale $<0.001$. Classic for COVID images were found to be in $100 \%$ and $94 \%$ patients respectively who had extremely elevated FDP and D-dimer level more than four times of UNL. Conclusion: HRCT chest in COVID-19 patients can detect severity of pulmonary involvement, thus had a major prognostic importance. Blood levels of FDP and D-dimer directly correlated with CT severity score and Classic CT images for COVID-19.

Key words: COVID-19, Computed tomography chest, D-dimer, Hypercoagulable state

\section{*Corresponding Author}

Dr. Govind Rankawat

E-mail id: govindrankawat@gmail.com

Copyright: This is an open-access article distributed under the terms of the Creative Commons Attribution license which permits unrestricted use, distribution, and reproduction in any medium for non-commercial use (Non Commercial, or CC-BY-NC) provided the original author and source are credited.

\section{INTRODUCTION}

Beginning in December, 2019, the rapid outbreak of corona virus disease 2019 COVID-19, which arose from severe acute respiratory syndrome corona virus 2 SARS-CoV-2 infection, has become a public health emergency of international concern. ${ }^{1}$ Although it is well documented that COVID-19 is primarily manifested as a respiratory tract infection, emerging data indicates that it should be regarded as a systemic disease involving multiple systems including cardiovascular, respiratory, gastrointestinal, neurological, hematopoietic and immune system..$^{2-4}$ On admission, many patients have reported as having at least one co morbidity with diabetes, hypertension, and cardiovascular and cerebro vascular diseases being most commonly reported conditions. ${ }^{5}$ Older people and those with co morbidities have high risk of COVID related mortality, but younger people without major underlying diseases may also present with potentially lethal complications such as fulminant myocarditis and disseminated intravascular coagulopathy DIC. ${ }^{6}$ In DIC fibrinolytic process predominated leads to degradation of fibrin clots by plasmin which

Volume-9| Issue-2 | $2020 \mid 9$ 
forms fibrin degradation product FDP and Ddimer. This mechanism may be a consequence of hyper coagulable state or venous thrombo embolism which is an emerging issue in COVID19 patients. ${ }^{7}$ The rate of symptomatic VTE in acutely ill hospitalized medical patients raises up to $10 \% .{ }^{8}$ Chest computed tomography $\mathrm{CT}$, in particular high-resolution computed tomography HRCT ,represents valuable tools in identifying patients with COVID-19 infections in an early stage when clinical symptoms may be unspecific or sparse. ${ }^{9,10}$ The purpose of our study was to evaluate results of FDP, D-dimer and HRCT characteristics in patients with COVID-19 infection retrospectively. We also aimed to explore the change in HRCT on a spectrum of range of FDP, D-dimer and whether there was a correlation between coagulation profile and imaging features in the course of the illness.

\section{MATERIALS AND METHODS}

Study Design: The present descriptive, retrospective analysis was done on 120 COVID19 positive patients admitted in S.M.S. Medical College Hospital, Jaipur, Rajasthan from 20th April to $15^{\text {th }}$ May 2020. Our institutional review board approved this retrospective study. All adult patients irrespective of their symptomatology were included in study with exclusion of patients who had incomplete records or critically ill. Informed consent was waived as the study involved no potential risk to patients. The data used was anonymized to protect the privacy and confidentiality of participants. Data Collection The diagnosis of COVID-19 was made based on the World Health Organization interim guidance, wherein confirmed cases denoted patients whose real-time reverse transcription-polymerase chain reaction rRT-PCR assay findings for nasal and pharyngeal swab specimens were positive for SARS-CoV-2 ${ }^{11}$.The epidemiological data was recorded inclusive of recent exposure history, clinical symptoms and signs, co morbidities was obtained.A detailed clinical evaluation was done. Routine biochemistry and hematologic evaluation were performed. To measure FDP and D-dimer $3 \mathrm{~mL}$ of venous blood sample drawn from COVID patients under aseptic condition and sent for quantitative value of sameby STA compact max machine. Review of CT images: All the patient sunder went initial high resolutions CTscan of chest with a dedicated CT machine while observing a proper disinfection protocol within an average of 4days of hospitalization. Thin section $1 \mathrm{~mm}$ CT images were acquire dona 128 slice Ingenia machine. The CT images were evaluated by a single radiologist who was blinded to the clinical condition of the patient to avoid bias, for the presence of ground glass haziness seen as increased attenuation with visible broncho-vascular markings, "crazypaving" ground glass opacities with interlobular thickening, consolidation increased attenuation of air space opacification. The distribution of lesions that is central versus peripheral, and anterior versus posterior was also noted. CT findings were overall classified as I Classic for COVID and II Negative for COVID. Classic findings include bilateral, peripheral GGOs with or without consolidation or crazy paving which were Typical for COVID, found frequently and specifically in COVID-19 pneumonia. Multifocal, diffuse, perihilar or unilateral GGOs which were Indeterminate for COVID-19 pneumonia arrive at a relatively confident radiological diagnosis also included in Classic for COVID CT images. Negative for COVID defined as radiologically negative CT images or those CT findings which reported to be uncommon or not occurring in COVID-19 pneumonia like lobar or segmental consolidation without GGOs or small nodules or cavitation or pleural effusion. ${ }^{12}$ The 3 lung lobes on the right and 2 lobes on the left were individually assessed and percentage involvement of the lobe was noted based on visual assessment. Visual severity scoring of CT chest was classified as Score-1 $<5 \%$ area involved, Score-2 5-25\% area involved, Score-3 25-50\% area involved, Score-4 50-75\% area involved, Score-5 $>75 \%$ area involved, making the total score 25 . A CT Severity Score was assigned out of 25 based on the percentage area involved in each of the 5 lobes. ${ }^{13}$ Statistical analysis The descriptive statistics for quantitative data was expressed as mean \pm standard deviation and qualitative data was expressed as $\mathrm{n} \%$. All the data was entered in SPSS software. For statistical analysis, students' $t$ test and chi square tests were used. The significance level was kept at $\mathrm{p}<0.05$

\section{RESULTS}

Data from COVID-19 positive patients were collected, evaluated, interpreted and correlated with each other to know severity of disease by their coagulation profile and radiological imaging in order to determine prognostic and diagnostic importance of HRCT chest. In our study group most of patients in fifth and sixth decades with mean age 50.40 years 95\% CI:50.40 \pm 3.59 , $\mathrm{SD}=16.39$.Females patients $40 \%$ were lesser than males $60 \%$ with an average sex ratio of female: male being 0.66 in our study. Out of total analyzed patients, $5949.16 \%$ were symptomatic while 61 patients $50.84 \%$ were asymptomatic. In present study $55 \%$ patients had some or other underlying co morbid disease in sample population. Percentage of comorbid patients in 
the study population were found as diabetes mellitus in $56 \%$, hypertension in $48.83 \%$, chronic obstructive pulmonary disease COPD /old kchest in $12 \%$, coronary artery disease in $9.32 \%$ and other diseases like hypothyroidism, anemia, CVA in $11.62 \%$. Table- 1

In our study population, coagulation abnormality determined by blood level of FDP and D-dimer. The mean value of FDP was found to be $17.9 \mu \mathrm{g} / \mathrm{mL} 95 \% \mathrm{CI}: 17.9 \pm 6.09, \mathrm{SD}=27.78$ and for d-dimer $3.3 \mu \mathrm{g} / \mathrm{mL} \quad 95 \%$ CI: $3.3 \pm 1.28$, $\mathrm{SD}=5.86 .69$ patients $58 \%$ patients had normal blood level of fibrin degradation product normal value $=0-5 \mu \mathrm{g} / \mathrm{mL}$ and28patients $23.33 \%$ had raised blood level of FDP upto four times of upper limits of normal ULN $6-20 \mu \mathrm{g} / \mathrm{mL}$ while23 patients $19 \%$ had elevated FDP level more than four times of ULN $>20 \mu \mathrm{g} / \mathrm{mL}$ graph 1 . Blood level of D- dimer was found to be normal $0-0.5 \mu \mathrm{g} / \mathrm{mL}$ in 54 patients $45 \%$,raised upto four times of ULN $0.5-2.0 \mu \mathrm{g} / \mathrm{mL}$ in 32 patients $26.66 \%$ and extremely raised more than four times of ULN $>2.0 \mu \mathrm{g} / \mathrm{mL}$ in 34 patients $28 \%$ graph 2 . Out of 120 patients, 76 were found to be radiologically positive on HRCT chest imaging while 44 patients $36.66 \%$ had normal or non-COVID CT findings. The lung involvement was evaluated according to HRCT imaging severity score with mean CT severity score were found to be $5.5595 \%$ CI: $5.5575 \pm 1.398$, $\mathrm{SD}=6.39$.We found that in our sample population 43 patients had CT severity score of zero. Among radiologically positive patients $44.73 \%$ patients had CT severity score between 1 to 5 figure $1,15.78 \%$ patients had CT severity score 6 to $10,21.05 \%$ patients had CT severity score 11 to $15,17.10 \%$ patients had CT severity score 16 to 20 and $3 \%$ patients had CT severity score 21 to 25 graph 3 , figure 2 . CT characteristics in COVID patients distributed as Classic for COVID figure 3 findings in $60.83 \%$ patients and $39.17 \%$ patients had Negative for COVID CT chest findings. Among radiologically positive patients $96.07 \%$ patients had Classic COVID findings on HRCT chest graph 4 . CT images of radiologically positive patients had isolated ground glass opacities GGOs in 50\%, isolated consolidation figure 5 in only $2.64 \%$ while remaining $47.6 \%$ patients had both GGOs and consolidation. Laboratory value of FDP and D-dimer were found to be higher in patients who had 'Classic for COVID' findings in CT images while it was lower in 'Negative for COVID' CT findings figure 4 .Patients who had value of FDP in normal range $0-5 \mu \mathrm{g} / \mathrm{mL}$, among them $42 \%$ patients had 'Classic for COVID' while 58\% patients found to be 'Negative for COVID' CT findings. Patients who had elevated FDP level up to four times of ULN, among them 75\% patients had 'Classic for COVID' and 25\% patients found to be 'Negative for COVID' CT findings. All the patients with very high FDP levels $>$ four times the ULN showed 'Classic for COVID' findings on chest CT. graph 5 .Patients who had normal value of D-dimer, among them $46.29 \%$ patients had 'Classic for COVID' while $53.71 \%$ patients found to be 'Negative for COVID' CT findings. Patients who had elevated D-dimer level up to four times of UNL, among them 50\% patients had 'Classic for COVID' and another $50 \%$ patients found to be 'Negative for COVID' CT findings. Patients who had extremely raised D-dimer level more than four times of ULN , among them $94.11 \%$ patients had 'Classic for COVID' while only $5.89 \%$ patients found to be 'Negative for COVID' CT findings graph 6 .

Pearson correlation coefficient $r$ between FDP and CT severity score were found $\mathrm{R}=+0.6494$ with $\mathrm{p}$-value for these variables were noted $\mathrm{p}<0.00001$.Patients who had normal value of FDP, among them $91.30 \%$ patients had CT severity score of $<10 / 25$ while remaining $8.70 \%$ patients had CT severity score $\geq 11 / 25$ with average CT severity score 2.6. Patients who had high level of FDP up to four times of ULN, among them $75 \%$ patients had CT severity score of $<10 / 25$ while remaining $25 \%$ patients had CT severity score $\geq 11 / 25$ with average CT severity score 4.6. Patients who had extreme level of FDP more than four times of ULN , among them $22 \%$ patients had CT severity score of $<10 / 25$ while $78 \%$ patients had CT severity score $\geq 11 / 25$ with average CT severity score 8.6 graph 7 .Pearson correlation coefficient $\mathrm{r}$ between D-dimer and CT severity score were found $\mathrm{R}=+0.7143$ with $\mathrm{p}$-value for these variables were noted $p<0.00001$.Patients who had normal value of $D$ dimer, among them $94.50 \%$ patients had CT severity score of $<10 / 25$ while remaining $5.50 \%$ patients had CT severity score $\geq 11 / 25$ with average CT severity score 3.22 . Patients who had high level of D-dimer up to four times of ULN, among them $84.4 \%$ patients had CT severity score of $<10 / 25$ while remaining $15.6 \%$ patients had CT severity score $\geq 11 / 25$ with average CT severity score 5.33. Patients who had extremely high level of D-dimer more than four times of ULN , among them $32 \%$ patients had CT severity score of $<10 / 25$ while $68 \%$ patients had CT severity score $\geq 11 / 25$ with average CT severity score 7.95 graph 8 . 
Table 1: Distribution of Epidemiological, Clinical features and Comorbid status of COVID-19 patients in study population

Characteristics

Number of
patients

Age Mean age 50.40 years $95 \%$ CI: $\mathbf{5 0 . 4 0} \pm 3.59$, $\mathrm{SD}=\mathbf{1 6 . 3 9}$

$<20$ year

$20-40$ year

40-60 year

$>60$ year

Gender

Male

Female

Clinical Presentation

Fever

Cough

SOB

Sore throat

Other

Comorbidity

Diabetes mellitus

Hypertension

COPD/K-chest

$\mathrm{CAD}$

Others

FDP Mean value $=17.9 \mu \mathrm{g} / \mathrm{mL} \quad 95 \%$ CI: $17.9 \pm 6.09, \mathrm{SD}=27.78$

$0-5.0 \mu \mathrm{g} / \mathrm{mL}$

$6.0-20.0 \mu \mathrm{g} / \mathrm{mL}$

$>20.0 \mu \mathrm{g} / \mathrm{mL}$

D-dimer Mean value $=3.3 \mu \mathrm{g} / \mathrm{mL} \quad 95 \%$ CI: $3.3 \pm 1.28, \mathrm{SD}=5.86$

$0-0.5 \mu \mathrm{g} / \mathrm{mL}$

$0.5-2.0 \mu \mathrm{g} / \mathrm{mL}$

$>2.0 \mu \mathrm{g} / \mathrm{mL}$

$\begin{array}{cc}\text { Percentage } & \text { Percentage Among } \\ \begin{array}{c}\text { Among Total } \\ \text { patients }\end{array} & \text { Affected patients }\end{array}$

$5.00 \%$

$22.50 \%$

$40 \%$

$32.50 \%$

$40 \%$

$60.00 \%$

$38 \%$

$35.83 \%$

$18 \%$

$9.16 \%$

$6.66 \%$

$78 \%$

$72.88 \%$

$36 \%$

$18.64 \%$

$13.55 \%$

$30 \%$

$25.83 \%$

$55 \%$

$46.96 \%$

$12 \%$

$9.09 \%$

$10.60 \%$
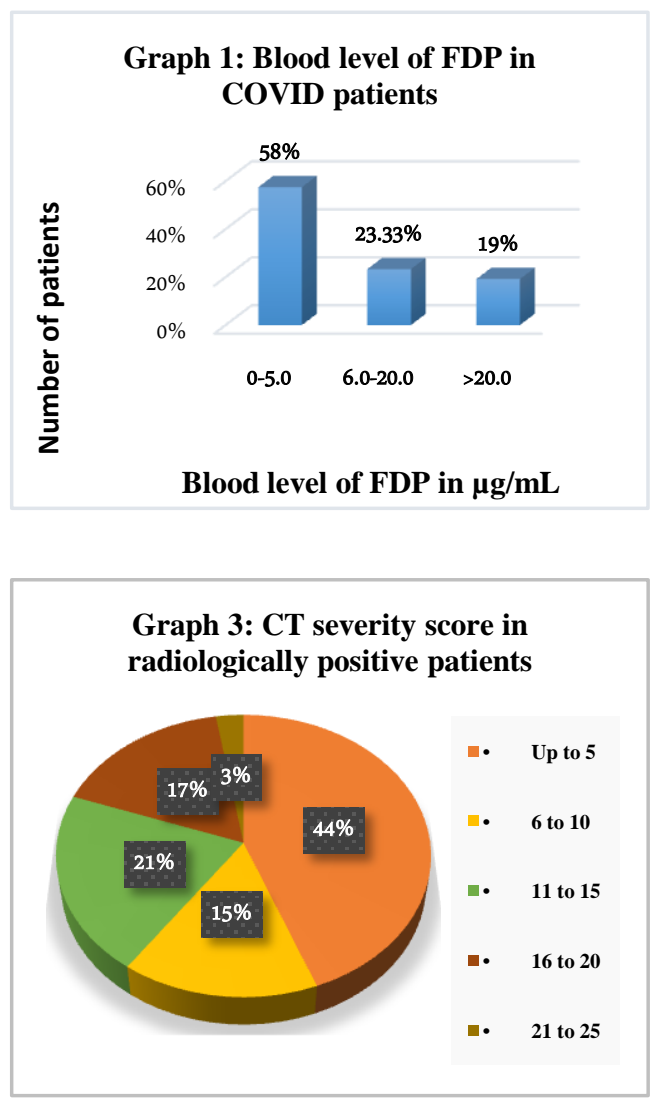

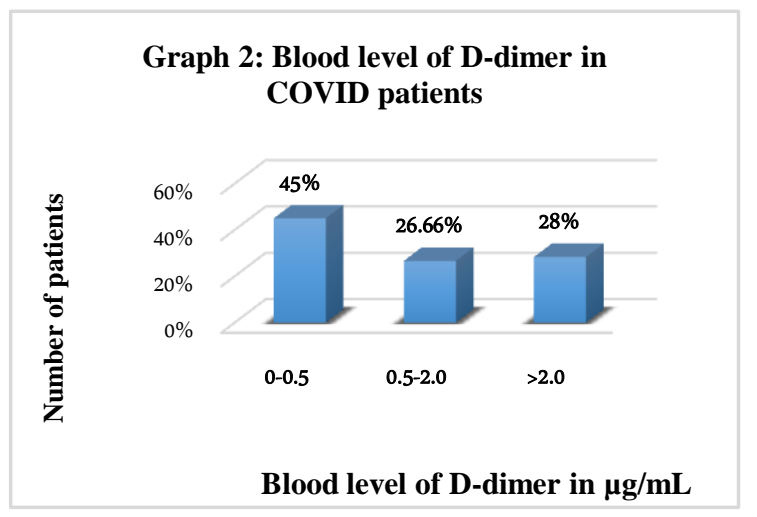

Graph 4: CT characteristic findings in COVID-19 patients

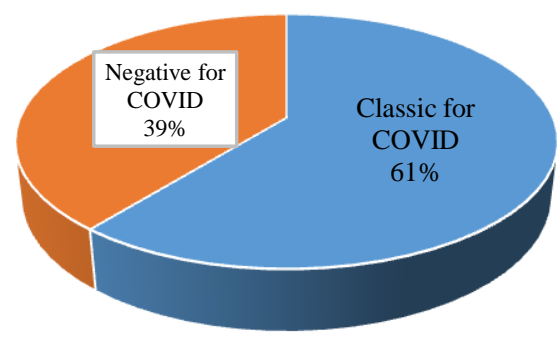

- Classic for COVID $\quad$ Negative for COVID 

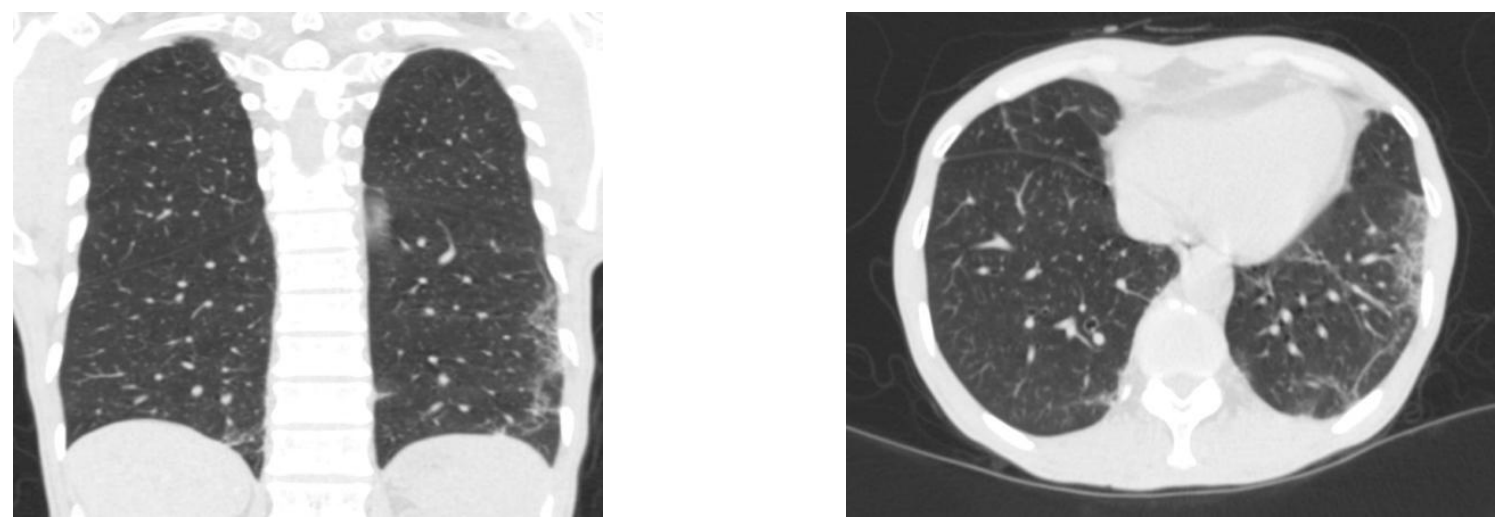

Figure 1: Axial and coronal sections of HRCT chest show bilateral, peripheral, lower lobe GGOs, crazy-paving and sub-pleural bands. Ct severity score in this patient was 5/25.

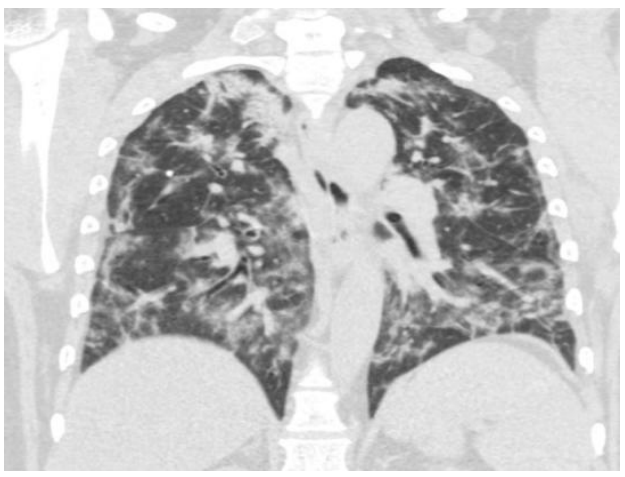

Figure 2: CT chest coronal section of a 38year old male patient showing involvement of all 5 lobes. The CT severity score in this patient was $23 / 25$.

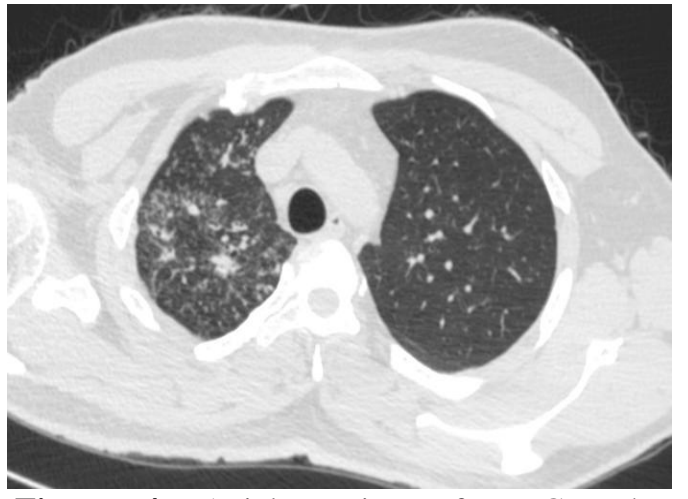

Figure 4: Axial section of HRCT chest showing calcified granuloma and multiple centrilobular nodules (tree-in-bud appearance), Negative for COVID CT findings.

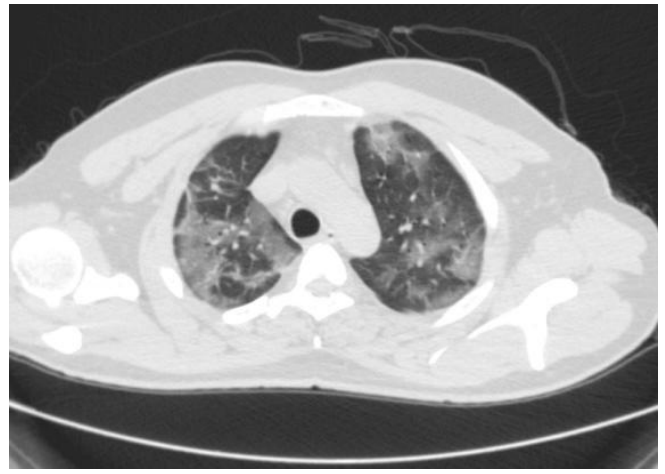

Figure 3: Axial section of HRCT chest in a 28year old male patient shows bilateral peripheral GGOs consistent with classic covid19 pneumonia features

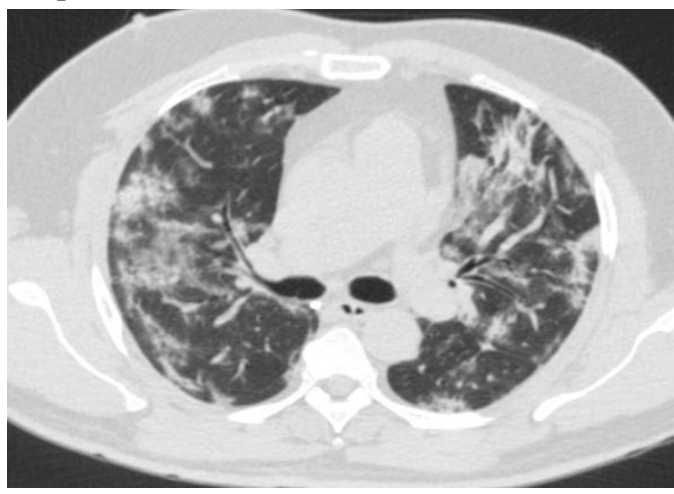

Figure 5: Axial section of CT chest of a 38year old male patient showing bilateral peripheral areas of consolidation. 

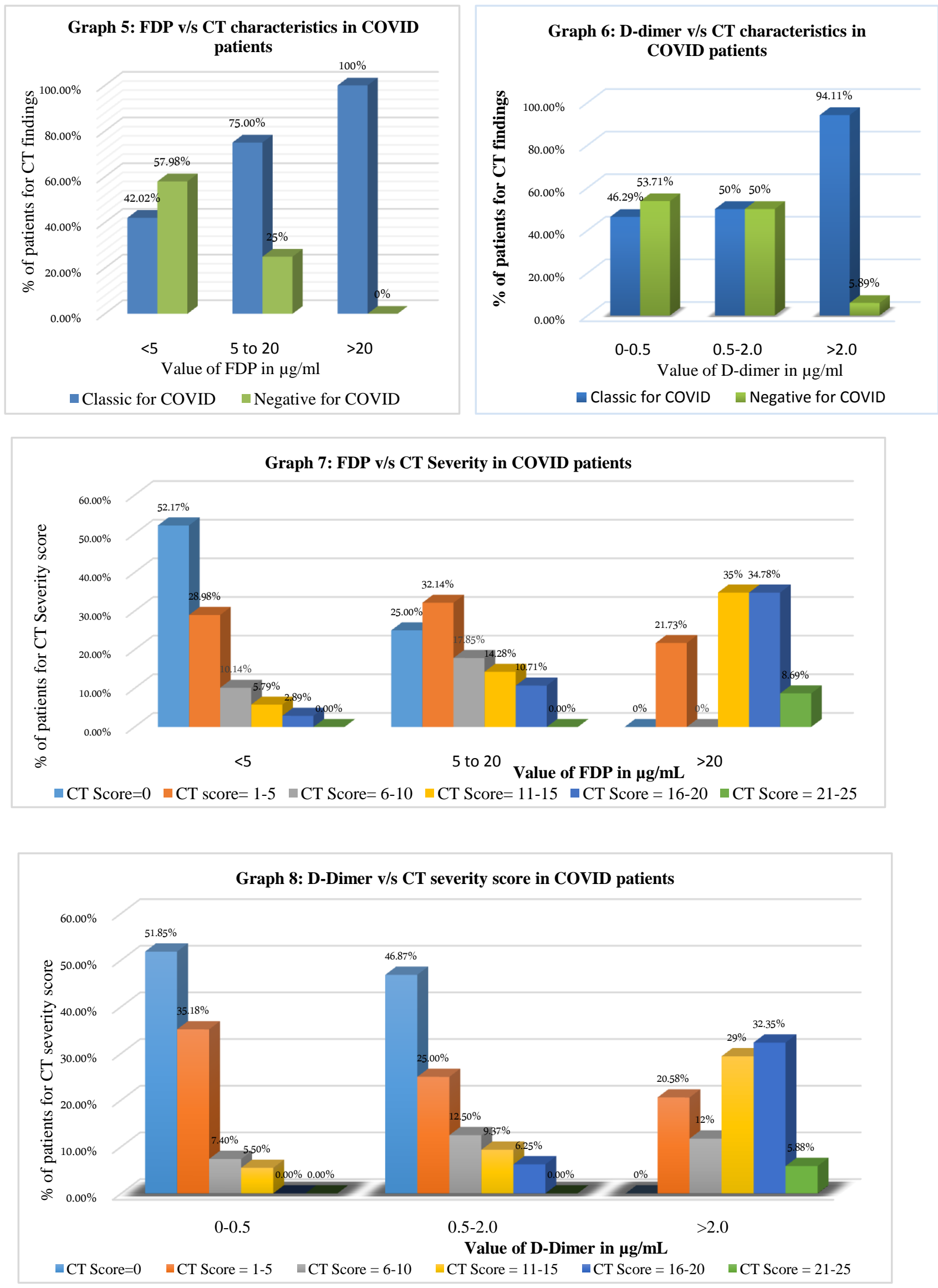

Volume-9 | Issue-2 | $2020 \mid 14$ 


\section{DISCUSSION}

Severe acute respiratory syndrome corona virus 2 SARS-CoV-2 causing corona virus disease 2019 COVID-19 has rapidly evolved from an epidemic outbreak in Wuhan, Chinal into a pandemic infecting more than one million individuals all over the world, whereas billions of citizens are affected by measures of social distancing and the socioeconomic impact of the pandemic. SARS-CoV-2 is mostly similar to SARS-CoV and invades host human cells by binding to the angiotens in-converting enzyme 2 ACE2 receptor. This binding to angiotens in-converting enzyme-2 ACE-2 receptor, which found in the heart, lungs, kidneys, and gastrointestinal tract ${ }^{14}$ thus facilitating viral entry into target cells. Although it is well documented that COVID-19 is primarily manifested as a respiratory tract infection, emerging data indicate that it should be regarded as a systemic disease involving multiple systems including cardiovascular, respiratory, gastrointestinal, neurological, hematopoietic and immune system. ${ }^{2-3}$ In the present study an attempt was made to outline distribution of age, gender, clinical features at presentation, co morbidity of patients, blood level of hyper coagulation parameters especially FDP and D-dimer, HRCT chest findings in COVID-19 patients, severity of patients on the basis of CT imaging and their correlation with FDP/D-dimer of patients to put diagnostic, therapeutic and prognostic tools for COVID-19 disease. Most adult patients to be afflicted have been observed to be in age range of 35 and 55 years. ${ }^{15}$ Some studies have reported an age distribution of adult patients between 25 and 89 years old. A study on early transmission dynamics of the virus has reported the median age of patients to be 59years, ranging from 15 to 89 years, with majority $59 \%$ patients affected being male. ${ }^{16}$ It has been suggested that population most at risk may be the people with poor immune function such as elderly and those with preexisting renal and hepatic dysfunction ${ }^{16}$. At present, severity of COVID patients is detected by their clinical manifestation, comorbid status, oxygen saturation, requirement of NIV or invasive ventilation but these parameters detect severity of COVID patients at later stages of the disease course. therefore, the biochemical tests which can be performed quickly and can easily detect coagulation abnormality, an important cause of morbidity and mortality in COVID patients. Coagulation

disordersarerelativelyfrequentlyencounteredam ongCOVID-19patients,especially among those with severe disease. ${ }^{17}$ HRCT chest imaging also an important prognostic tool to detect the extent and severity of COVID-19pneumonia and can be correlated with abnormal levels of the indicators of altered coagulation profile. A total of 120 patients were analyzed along the course of the study. Most of COVID-19 patients of our study group in SMS Medical College Hospital, Jaipur, Rajasthan were in their fifth to sixth decades of life with mean age of 50.40 year and male gender was affected more as compared to females, with an average sex ratio being 0.66.In symptomatic patients, fever and cough were the most common presenting features, followed by shortness of breath, sore throat and headache while few patients also presented with chest pain and non-respiratory symptoms like pain abdomen, fatigue, joints pain, altered sensorium, etc. In our study nearly one third of asymptomatic patients were found to be having abnormal chest CT scans. These asymptomatic COVID-19 patients may act as potential carrier for transmission of virus in the society, and pose a real diagnostic and containment challenge for health care professionals. In the present study population $54 \%$ patients had underlying comorbid disease with multiple comorbid diseases being was more prevalent. The most prevalent comorbidity observed in present study was Diabetes mellitus in followed by Hypertension, Chronic obstructive pulmonary disease COPD /Old K-chest, Coronary artery disease CAD and other diseases like hypothyroidism, anemia, CVA etc.

Nearly two fifth patients had raised blood level of FDP while more than half of patients had elevated blood level of D-dimer. Proportional extreme value of D-dimer was found to be higher in $28 \%$ patients compared to extreme value of FDP in 19\% patients in COVID-19 patients. Tang et al. reported on abnormal coagulation parameters and poor prognosis in 183 consecutive patients with Covid-19 pneumonia. ${ }^{17}$ Those who did not survive their illness compared with survivors had higher D-dimer levels, fibrin degradation products FDP.

The prominent radiological feature of COVID-19 is bilateral ground glass opacity in the chest CT scans ${ }^{18}$. In present study most of radiologically positive patients $96 \%$ had Classic for COVID images on HRCT chest which includes GGO in bilateral, peripheral, diffuse and lower lobe predominance distribution of opacities with few of them deviated towards unilaterality and lobar distribution. Laboratory value of FDP and Ddimer were found to be higher in patients who had Classic for COVID findings in CT images while it was lower in Negative for COVID CT findings. Less than half of patients in $42 \%$ patients were found to be Classic for COVID on CT chest in patients who had normal blood level of FDP while three fourth patients had Classic 
for COVID on CT chest in patients who had high value of FDP up to four times of ULN. Surprisingly all patients were found to be Classic for COVID on CT chest in patients who had extremely high value of FDP more than four times of ULN, Similarly less than half of patients in $46 \%$ patients were found to be Classic for COVID on CT chest in patients who had normal blood level of D-dimer while half patients had Classic for COVID on CT chest in patients who had high value of D-dimer up to four times of ULN. Most of patients 94\% were found to be Classic for COVID on CT chest in patients who had extremely high value of D-dimer more than four times of ULN. Hence value of FDP and Ddimer directly proportional to Classic COVID findings on CT images. As value of FDP and Ddimer raised simultaneously characteristics CT findings for various COVID lesions including GGOs, consolidation, subpleural sparing etc. also raised suggesting poor prognosis of patients and require more therapeutic intervention. For evaluation of severity of lung involvement on HRCT chest a CT severity score formulated starting from 0 to 25 in number and categorized in six groups. Nearly one third COVID patients $35.8 \%$ patients had normal $\mathrm{CT}$ images and categorized as radiologically negative with CT severity score of zero. In radiologically positive patients nearly three fifth patients $60.51 \%$ had CT severity score $<10 / 25$ while remaining had score of $>10 / 25$. As per clinical spectrum of our study group CT severity score also found to be in lower range in most of patients. Blood level of FDP and D-dimer also correlated with CT severity score and we found proportional CT severity score was found to be higher in patients who had raised blood level of FDP and D-dimer. Majority of patients $78 \%$ patients had CT severity score $>10 / 25$ in patients who had blood level of FDP extremely high More than four times of ULN while proportion of patients reduced $25 \%$ patients for CT severity score $>10 / 25$ in patients who had high level of FDP up to four times of ULN and only few patients $8.70 \%$ patients had CT severity score $>10 / 25$ in patients who had normal blood level of FDP. Similarly more than two third patients $68 \%$ had CT severity score $>10 / 25$ in patients who had extremely high value of Ddimer more than four times of ULN while only $15.6 \%$ patients had CT severity score $>10 / 25$ in patients who had high value of D-dimer up to four times of ULN. A few patients only 5.5\% patients had CT severity score $>10 / 25$ among who had normal blood level of D-dimer. A positive pearson correlation coefficient were noted between FDP/D-dimer and CT severity score with p-value $<0.00001$. In this study, we assessed the involvement of lungs with $\mathrm{CT}$ chest images, in which nearly two third patients $63.33 \%$ patients had positive CT findings while less than half of patients $49.16 \%$ were symptomatic.

We scored the chest CT imaging and found thathigher CT imaging score were found in patients who had raised blood level of FDP and D-dimer while CT imaging score were found to be lower in patients who had normal value of FDP and D-dimer. These data suggested that CT imaging score with value of FDP and D-dimer may be an informative indicator to predict the severity of the disease. As CT findings correlated with hyper coagulable state, showing a higher CT severity score in clinically worse patients. Thus, the percentage involvement of lung and CT severity score can help prognosticate and tailor the clinical management of patients.

\section{CONCLUSION}

COVID-19 infection presented with fever, cough, shortness of breath, sore throat etc. Diabetes mellitus, hypertension, COPD/K-Chest and CAD were found as major comorbid condition. COVID patients had raised blood level of FDP and D-dimer suggesting hyper coagulable state. CT severity score of COVID patients directly correlated with blood level of FDP and D-dimer of patients. The results of this study confirmed that chest CT is important in the management and prognostication of the COVID-19infection. Despite meticulous treatments, most patients demonstrated progressions in the early stage from illness onset, according to the follow-up CT examinations. Our clinical findings show that radiological features positively correlate with these verity of lung abnormalities quantified on initial CT. Being familiarized with the clinical and CT features and theearly changes of the COVID-19 infection is of paramount importance.

Conflicts of interest: Declared, authours have no conflict of interest

Funding/Financial support: None

Acknowledgments: I would like to thanks the anonymous referees for their useful suggestion. I would like to thanks to my professionals Dr. Vishal Gupta, Dr. C. L. Nawal, Dr. S. Banerjee, Dr. Prakash Keswani, Dr. Sunil Mahavar, Dr. R S Chejara, Dr. Vidyadhar Singh, Dr. Amitabh Dube, Dr. Shivankan Kakkar, Dr. Kapil Choudhary, Department of General Medicine SMS Medical college and attached group of Hospital, Jaipur for their valuable support in patients care and Department of Radiodiagnosis for providing radiological information of COVID-19 patients. 


\section{REFERENCES}

1. WHO main website. https://www.who.int accessed March 10th, 2020

2. Driggin E, Madhavan MV, Bikdeli B, et al. Cardiovascular Considerations for Patients, Health Care Workers, and Health Systems During the corona virus Disease 2019 COVID-19 Pandemic. J Am Coll Cardiol 2020 Mar 18.

3. Bangash MN, Patel J, Parekh D. COVID-19 and the liver: little cause for concern. Lancet Gastroenterol Hepatol 2020 Mar 20.

4. Mehta P, McAuley DF, Brown M, et al. COVID-19: consider cytokine storm syndromes and immunosuppression. Lancet 2020 Mar 28; 395 10229 : 1033-1034.

5. Chen N, Zhou M, Dong $X$, et al. Epidemiological and clinical characteristics of 99 cases of 2019 novel corona virus pneumonia in Wuhan, China: a descriptive study. Lancet. 2020. doi: 10.1016/S0140$67362030211-7$

6. Tang N, Li D, Wang X, Sun Z. Abnormal coagulation parameters are associated with poor prognosis in patients with novel corona virus pneumonia. J Thromb Haemost 2020 Apr; $184: 844-847$.

7. Llitjos JF, Leclerc M, Chochois C, Monsallier JM, Ramakers M, Auvray M, Merouani K.J Thromb Haemost. 2020 Apr 22. doi: $10.1111 /$ jth.14869. Online ahead of print.PMID: 32320517

8. Kahn SR, Lim W, Dunn AS, et al. Prevention of VTE in nonsurgical patients: Antithrombotic Therapy and Prevention of Thrombosis, 9th ed: American College of Chest Physicians Evidence-Based Clinical Practice Guidelines. Chest 2012 Feb; 1412 Suppl : e195S-e226S.

9. FangY,ZhangH,XieJ,etal.SensitivityofchestC TforCOVID-19:comparison to RT-PCR. Radiology.2020;200432.

10. XieX,ZhongZ,Zhao etal.ChestCTfortypical2019-

nCoVpneumonia:re- lationship to negative RT-PCR testing. Radiology.2020;200343.

11. Li Q, Guan X, Wu P, Wang X, Zhou L, Tong $\mathrm{Y}$, et al. Early transmission dynamics in Wuhan, China, of novel corona virusinfected pneumonia. N Engl J Med. 2020. https://doi.org/10.1056/NEJMoa2001316 [Epub ahead of print].

12. simpson S, Kay FU et al. Radiological Society of North America Expert Consensus Statement on Reporting Chest CT Findings Related to COVID-19. Endorsed by the Society of Thoracic Radiology, the American College of Radiology. Radiology: cardiothoracic imaging.

13. Bernheim A, Mei X, Huang $M$ et al. Chest CT Findings in corona virus Disease-19 COVID-19 : Relationship to Duration of Infection. Radiology. 2020:200463.

14. Ksiazek, T.G.; Erdman, D.; Goldsmith, C.S.; Zaki, S.R.; Peret, T.; Emery, S.; Tong, S.; Urbani, C.; Comer, J.A.; Lim, W.; et al. A Novel corona virus Associated with Severe Acute Respiratory Syndrome. N. Engl. J. Med. 2003, 348, 1953-1966. [CrossRef] [PubMed]

15. Medical expert group of Tongji hospital. Quick guide to the diagnosis and treatment of pneumonia for novel corona virus infections third edition . Herald Med. 2020. http://kns.cnki.net/kcms/detail/42.1293.r.202 00130.1803. 002.html. Accessed 2 Feb 2020.

16. Li Q, Guan X, Wu P, Wang X, Zhou L, Tong $\mathrm{Y}$, et al. Early transmission dynamics in Wuhan, China, of novel corona virusinfected pneumonia. N Engl J Med. 2020. https://doi.org/10.1056/NEJMoa2001316.

17. Tang N, Li D, Wang X, Sun Z. Abnormal coagulation parameters are associated with poor prognosis in patients with novel corona virus pneumonia. J Thromb Haemost JTH. 2020;18:844-847.

18. Pan F, Ye T, Sun P, Gui S, Liang B, Li L, Zheng D, Wang J, Hesketh RL, Yang L and Zheng C. Time Course of Lung Changes On Chest CT During Recovery From 2019 Novel corona virus COVID-19 Pneumonia. Radiology.2020:200370. 